\title{
The Iwasawa Invariants and the Higher $K$-Groups Associated to Real Quadratic Fields
}

\author{
Hiroki Sumida-Takahashi
}

\section{CONTENTS}

\section{Introduction}

2. Notation and Conjectures

3. Iwasawa Invariants of $\mathbb{Q}\left(\sqrt{f_{\chi}}, \zeta_{p}\right)$

4. Higher $K$-Groups of the Ring of Integers of $\mathbb{Q}\left(\sqrt{f_{\chi}}\right)$

5. Algorithms for Computing Arithmetic Elements

Acknowledgments

References

2000 AMS Subject Classification: Primary 11R23, 11R70

Keywords: Iwasawa invariant, $K$-group, Vandiver's conjecture, Greenberg's conjecture
Using fast algorithms, we compute the Iwasawa invariants of $\mathbb{Q}\left(\sqrt{f}, \zeta_{p}\right)$ in the range $1<f<200$ and $3 \leq p<100,000$. From these computational results, we obtain concrete information on the higher $K$-groups of the ring of integers of $\mathbb{Q}(\sqrt{f})$.

\section{INTRODUCTION}

Let $\chi$ be an even Dirichlet character of conductor $f=f_{\chi}$. The generalized Bernoulli numbers $B_{k, \chi}$ are defined by

$$
\sum_{a=1}^{f} \frac{\chi(a) t e^{a t}}{e^{f t}-1}=\sum_{k=0}^{\infty} B_{k, \chi} \frac{t^{k}}{k !}
$$

First, let us look back over the case of $\chi=\chi^{0}$ the trivial character. For $k \neq 1, B_{k, \chi^{0}}$ is the $k$ th Bernoulli number $B_{k}$, and $B_{1, \chi^{0}}=B_{1}+1=1 / 2$. A pair of integers $(p, k)$ is said to be an irregular pair if $p$ is a prime, $k$ is an even integer satisfying $2 \leq k \leq p-3$, and $p$ divides the numerator of $B_{k}=B_{k, \chi^{0}}$. Irregular pairs have been computed by Kummer, Vandiver, D.H. Lehmer, E. Lehmer, Selfridge, Nicol, Pollack, Johnson, Wada, Wagstaff, Tanner, Ernvall, Metsänkylä, Buhler, Crandall, Sompolski, and Shokrollahi. These computations were originally used to verify Fermat's Last Theorem. However, even after the proof was completed by Wiles, these computations are still important because they give us concrete knowledge of the ideal class group of cyclotomic fields.

Let $p$ be an odd prime number and $A_{n}$ the $p$-part of the ideal class group of $K_{n}=\mathbb{Q}\left(\zeta_{p^{n+1}}\right)$. Let $\omega=\omega_{p}$ be the Teichmüller character $\mathbb{Z} / p \mathbb{Z} \rightarrow \mathbb{Z}_{p}$ such that $\omega(a) \equiv$ $a \bmod p$. We identify $\Delta=\operatorname{Gal}\left(K_{\infty} / \mathbb{Q}_{\infty}\right)$ with $(\mathbb{Z} / p \mathbb{Z})^{\times}$. Let

$$
e_{\omega^{k}}=\frac{1}{\sharp \Delta} \sum_{\delta \in \Delta} \omega^{k}(\delta) \delta^{-1}
$$

be the idempotent of the group ring $\mathbb{Q}_{p}[\Delta]$. Then we have

$$
A_{n}=\bigoplus_{k: \text { even }} e_{\omega^{k}} A_{n} \oplus \bigoplus_{p-k: \text { odd }} e_{\omega^{p-k}} A_{n}
$$

(c) A K Peters, Ltd. $1058-6458 / 2005 \$ 0.50$ per page Experimental Mathematics 14:3, page 307 
where $k$ is an even integer with $2 \leq k \leq p-1$. We denote the even part (respectively odd part) by $A_{n}^{+}$(respectively $A_{n}^{-}$). Let $r_{p}$ be the irregularity index, i.e., the number of irregular pairs $(p, k)$. For any prime number $p<12,000,000$, it has been verified that

$$
A_{n}^{+}=\{0\} \text { and } A_{n}^{-} \simeq\left(\mathbb{Z} / p^{n+1} \mathbb{Z}\right)^{r_{p}} \text { for all } n \geq 0
$$

(see [Buhler et al. 93] and [Buhler et al. 01]). The former statement is called Vandiver's conjecture. We have a naive explanation for the fact that we have not been able to find any counterexample. If we follow the argument of [Washington 97, pages 158-159], we can expect that the number of exceptions to Vandiver's conjecture for $x_{0}<p \leq x_{1}$ is approximately $\left(\log \log x_{1}-\log \log x_{0}\right) / 2$, and $(\log \log 12,000,000-\log \log 37) / 2=0.7536143467 \ldots$ is perhaps too small to find a counterexample. However, many number theorists may doubt the above expected number. As a matter of fact, we have to consider some effects on ideal class groups from an upper bound for the numerators of Bernoulli numbers, and from a lower bound for discriminants (see [Washington 97, pages 221230]). If there is another strong bound, the actual number could be much less than the above number.

In this paper, following [Sumida-Takahashi 04], we consider the $\chi \omega^{k}$-part instead of the $\omega^{k}$-part, where $\chi$ is an even quadratic Dirichlet character. We consider quadratic characters, because their values, as well as the trivial character, are included in $\mathbb{Q}$. The first main purpose of this paper is to effectively find "exceptional pairs" $\left(p, \chi \omega^{k}\right)$ in order to use them to discuss the expected number. Here, we call $\left(p, \chi \omega^{k}\right)$ an exceptional pair if and only if $\chi \omega^{k}(p) \neq 1, \chi \omega^{1-k}(p) \neq$ 1 , and one of the following conditions is satisfied: $\nu_{p}\left(\chi \omega^{k}\right)>0, \quad v_{p}\left(L_{p}\left(1, \chi \omega^{k}\right)\right)>1, \quad v_{p}\left(L_{p}\left(0, \chi \omega^{k}\right)\right)>$ 1 , or $\tilde{\lambda}_{p}\left(\chi \omega^{k}\right)>1$, where $\nu_{p}\left(\chi \omega^{k}\right)$ is the $\chi \omega^{k}$-part of $\nu_{p}$-invariant and $v_{p}$ is the $p$-adic valuation such that $v_{p}(p)=1$ (see Section 3 for the details). We actually computed the Iwasawa invariants of $\mathbb{Q}\left(\sqrt{f_{\chi}}, \zeta_{p}\right)$ in the range $1<f_{\chi}<200$ and $3 \leq p<100,000$. From our data, the actual number of exceptional pairs seems to be near to the expected number in the range. On the other hand, we could not find any exceptional pair for $f_{\chi}=5$ and $p<2,000,000$ nor for the trivial character.

Let $F=F_{\chi}$ be the real quadratic field associated to $\chi$ and $\mathcal{O}_{F}$ the ring of integers of $F$. By [Kahn 93] and [Kolster et al. 96], there are relations between Quillen's $K$-groups $K_{n}\left(\mathcal{O}_{F}\right)$ and the Iwasawa modules for unramified abelian $p$-extensions of $\cup_{n \geq 0} F\left(\zeta_{p^{n}}\right)$. The second main purpose of this paper is to give concrete information on the higher $K$-groups of $\mathcal{O}_{F}$ by using the computational results. For example, we found that for $3 \leq p<100,000, p$ divides the order of $K_{68372}\left(\mathcal{O}_{\mathbb{Q}(\sqrt{8})}\right)$ if and only if $p=34,301$ under the Quillen-Lichtenbaum conjecture.

\section{NOTATION AND CONJECTURES}

In this section, we introduce some conjectures on higher $K$-groups and Iwasawa modules.

Let $F$ be a finite extension of $\mathbb{Q}$. The following theorems and conjecture are well known.

Theorem 2.1. (Quillen.) For all $n \geq 0, K_{n}\left(\mathcal{O}_{F}\right)$ is a finitely generated $\mathbb{Z}$-module.

Theorem 2.2. (Borel.) For $m \geq 1$,

$$
\operatorname{rank}_{\mathbb{Z}}\left(K_{2 m-1}\left(\mathcal{O}_{F}\right)\right)= \begin{cases}r_{1}(F)+r_{2}(F) & \text { if } m \text { is odd, } \\ r_{2}(F) & \text { if } m \text { is even, }\end{cases}
$$

where $r_{1}(F)$ is the number of real embeddings of $F$, and $r_{2}(F)$ is the number of pairs of complex embeddings of $F$. Further,

$$
K_{2 m-2}\left(\mathcal{O}_{F}\right) \text { is finite. }
$$

\section{Conjecture 2.3. (The Quillen-Lichtenbaum Conjecture.)} The natural map (via p-adic Chern characters)

$$
K_{2 m-i}\left(\mathcal{O}_{F}\right) \otimes \mathbb{Z}_{p} \rightarrow H_{e ́ t}^{i}\left(\operatorname{Spec}\left(\mathcal{O}_{F}[1 / p]\right), \mathbb{Z}_{p}(m)\right)
$$

is an isomorphism for all $m \geq 2, i=1,2$, and any odd prime number $p$, where $A(m)$ is the mth Tate twist of a Galois module A.

The surjectivity of $p$-adic Chern characters was proved in [Soulé 79, Dwyer and Friedlander 85, Kurihara 92, Kahn 93]. For $p=2$, we need to modify the statement. Voevodsky's work on Milnor's conjecture resolved the conjecture for $p=2$ (see [Voevodsky 03]). We simply denote $H_{\text {ét }}^{i}\left(\operatorname{Spec}\left(\mathcal{O}_{F}[1 / p]\right), A\right)$ by $H^{i}\left(\mathcal{O}_{F}, A\right)$. Let $K=$ $F\left(\zeta_{p}\right)$ and denote by $K_{\infty}$ the cyclotomic $\mathbb{Z}_{p}$-extension of $K$. Let $G_{\infty}=\operatorname{Gal}\left(K_{\infty} / F\right), \Delta=\operatorname{Gal}\left(K_{\infty} / F_{\infty}\right)$, and $\Gamma=\operatorname{Gal}\left(K_{\infty} / K\right)$. Then we have $G_{\infty}=\Delta \times \Gamma$. Let $L_{\infty}$ be the maximal unramified abelian $p$-extension of $K_{\infty}$ and $L_{\infty}^{\prime}$ the maximal unramified abelian $p$-extension of $K_{\infty}$ in which every prime divisor lying above $p$ splits completely. Let $X_{\infty}=\operatorname{Gal}\left(L_{\infty} / K_{\infty}\right)$ and $X_{\infty}^{\prime}=\operatorname{Gal}\left(L_{\infty}^{\prime} / K_{\infty}\right)$. 
Theorem 2.4. [Kolster et al. 96, Section 3, Section 4] For $m \neq 0$, we have

$$
H^{1}\left(\mathcal{O}_{F}, \mathbb{Z}_{p}(m)\right)_{\text {tors }} \simeq H^{0}\left(\mathcal{O}_{F}, \mathbb{Q}_{p} / \mathbb{Z}_{p}(m)\right) .
$$

For $m \neq 1$, we have an exact sequence

$$
\begin{aligned}
0 \rightarrow X_{\infty}^{\prime}(m-1)_{G_{\infty}} & \rightarrow H^{2}\left(\mathcal{O}_{F}, \mathbb{Z}_{p}(m)\right) \\
& \rightarrow \prod_{v \mid p} H^{2}\left(F_{v}, \mathbb{Z}_{p}(m)\right) \\
& \rightarrow H^{0}\left(\mathcal{O}_{F}, \mathbb{Q}_{p} / \mathbb{Z}_{p}(1-m)\right)^{\vee} \\
& \rightarrow 0,
\end{aligned}
$$

where $A^{\vee}=\operatorname{Hom}_{\mathbb{Z}_{p}}\left(A, \mathbb{Q}_{p} / \mathbb{Z}_{p}\right)$.

It is not difficult to compute $H^{0}\left(\mathcal{O}_{F}, \mathbb{Q}_{p} / \mathbb{Z}_{p}(m)\right)$ and $H^{2}\left(F_{v}, \mathbb{Z}_{p}(m)\right)$ (see Section 4$)$. Assume that the QuillenLichtenbaum conjecture is true. Then, by Theorem 2.2 and Theorem 2.4, it is not difficult to determine the structure of $K_{2 m-1}\left(\mathcal{O}_{F}\right)$ as an abelian group. Further, by the exact sequence in Theorem 2.4, we can obtain the order of $K_{2 m-2}\left(\mathcal{O}_{F}\right)$ by using the order of $X_{\infty}^{\prime}(m-1)_{G_{\infty}}$. Let us consider the following case:

$$
F \text { is totally real and } F \cap \mathbb{Q}\left(\zeta_{p}\right)=\mathbb{Q} \text {. }
$$

For a $\mathbb{Z}_{p}[\Delta]$-module $A$ and a character $\omega^{m}$ of $\Delta \simeq$ $\operatorname{Gal}\left(\mathbb{Q}\left(\zeta_{p}\right) / \mathbb{Q}\right)$, we denote $e_{\omega^{m}} A$ by $A^{(m)}$. Since

$$
X_{\infty}^{\prime}(m-1)_{G_{\infty}} \simeq\left(X_{\infty}^{\prime(1-m)} \otimes \mathbb{Z}_{p}(m-1)\right)_{\Gamma},
$$

it is important to study the structure of ${X^{\prime}}_{\infty}^{(1-m)}$ as an Iwasawa module. If $m$ is even, $X_{\infty}^{\prime(1-m)}$ has no nontrivial finite submodule (see [Washington 97, page 290]). Therefore, the order of the $\Gamma$-coinvariant quotient can be obtained from the Iwasawa polynomial for $X_{\infty}^{\prime(1-m)}$. By Iwasawa's main conjecture, proved by [Mazur and Wiles 84] and [Wiles 90], the polynomial is essentially the $p$-adic $L$-function. Therefore, if $F$ is abelian, it suffices to compute the Kubota-Leopoldt $p$-adic $L$-function. On the other hand, if $m$ is odd, it seems to be more

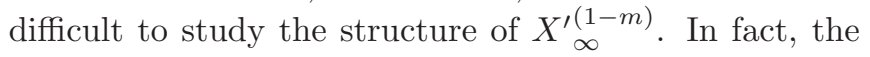
following classical conjectures are still open.

\section{Conjecture 2.5. (Vandiver's Conjecture.) $\quad$ For $F=\mathbb{Q}$} and any odd integer $m,{X_{\infty}^{\prime}}_{\infty}^{(1-m)}$ is trivial.

Conjecture 2.6. (Greenberg's Conjecture.) For any totally real number field $F$ and any odd integer $m, X_{\infty}^{\prime(1-m)}$ is finite.

So far we have not been able to find any counterexample to the conjectures. Conjecture 2.5 has been verified for all $p<12,000,000$. Conjecture 2.6 has mainly been verified for real abelian fields with small discriminants and some prime numbers $p=3,5,7, \ldots$ by using cyclotomic units and auxiliary prime numbers (see [Ichimura and Sumida 96] and [Kraft and Schoof 95]). In [SumidaTakahashi 04], the author exploited a method to effectively check the exact value of the $p$-part of the class number by using Gauss sums and auxiliary prime numbers. We will give some numerical examples of the Iwasawa invariants and the higher $K$-groups in the following sections.

\section{IWASAWA INVARIANTS OF $\mathbb{Q}\left(\sqrt{f_{\chi}}, \zeta_{p}\right)$}

Let $\chi$ be an even quadratic Dirichlet character and $p$ an odd prime number. Set $F=F_{\chi}=\mathbb{Q}\left(\sqrt{f_{\chi}}\right)$ and $K=$ $\mathbb{Q}\left(\sqrt{f_{\chi}}, \zeta_{p}\right)$. We use the notation in the previous sections. We set $\Delta^{\prime}=\operatorname{Gal}\left(K_{\infty} / \mathbb{Q}_{\infty}\right)$ and $e_{\psi}^{\prime}=\frac{1}{\sharp \Delta^{\prime}} \sum_{\delta \in \Delta^{\prime}} \psi(\delta) \delta^{-1}$ for a Dirichlet character $\psi$ of $\Delta^{\prime}$. For a $\mathbb{Z}_{p}\left[\Delta^{\prime}\right]$-module $A$, we denote $e^{\prime}{ }_{\psi} A$ by $A^{\psi}$. Let $\lambda_{p}(\psi), \mu_{p}(\psi)$, and $\nu_{p}(\psi)$ (respectively $\lambda_{p}^{\prime}(\psi), \mu_{p}^{\prime}(\psi)$, and $\nu_{p}^{\prime}(\psi)$ ) be the Iwasawa invariants associated to $X_{\infty}^{\psi}$ (respectively $X_{\infty}^{\prime}{ }_{\infty}$ ), i.e.,

$$
\begin{aligned}
\sharp A_{n}^{\psi} & =p^{\lambda_{p}(\psi) n+\mu_{p}(\psi) p^{n}+\nu_{p}(\psi)} \\
\text { (respectively } \sharp A_{n}^{\prime \psi} & =p^{\lambda_{p}^{\prime}(\psi) n+\mu_{p}^{\prime}(\psi) p^{n}+\nu_{p}^{\prime}(\psi)} \text { ) }
\end{aligned}
$$

for sufficiently large $n$. By Ferrero-Washington's theorem, we have $\mu_{p}(\psi)=\mu_{p}^{\prime}(\psi)=0$ for all $p$ and $\psi$.

Assume that $\psi$ is even. The Iwasawa polynomial $g_{\psi}(T) \in \mathbb{Z}_{p}[T]$ for the $p$-adic $L$-function is defined as follows. Let $L_{p}(s, \psi)$ be the $p$-adic $L$-function constructed by [Kubota and Leopoldt 64 ]. Let $f_{0}$ be the least common multiple of $f_{\psi}$ and $p$. By [Iwasawa $72, \S 6$ ], there uniquely exists $G_{\psi}(T) \in \mathbb{Z}_{p}[[T]]$ satisfying $G_{\psi}\left(\left(1+f_{0}\right)^{1-s}-1\right)=$ $L_{p}(s, \psi)$ for all $s \in \mathbb{Z}_{p}$ if $\psi \neq \chi^{0}$. By [Ferrero and Washington 79], it was proved that $p$ does not divide $G_{\psi}(T)$. Therefore, by the $p$-adic Weierstrass preparation theorem, we can uniquely write $G_{\psi}(T)=g_{\psi}(T) u_{\psi}(T)$, where $g_{\psi}(T)$ is a distinguished polynomial of $\mathbb{Z}_{p}[T]$ and $u_{\psi}(T)$ is an invertible element of $\mathbb{Z}_{p}[[T]]$. Let $\tilde{\lambda}_{p}(\psi)=\operatorname{deg} g_{\psi}(T)$.

Let $k$ be an even integer with $2 \leq k \leq p-3$. Then $\chi \omega^{k}$ is an even character. For a pair $\left(p, \chi \omega^{k}\right)$, we set the following condition:

$$
\chi \omega^{k}(p) \neq 1 \text { and } \chi \omega^{1-k}(p) \neq 1 .
$$

If $\chi \omega^{k}(p) \neq 1$, we have $\lambda_{p}\left(\chi \omega^{k}\right)=\lambda_{p}^{\prime}\left(\chi \omega^{k}\right)$ and $\nu_{p}\left(\chi \omega^{k}\right)=\nu_{p}^{\prime}\left(\chi \omega^{k}\right)$. In the range $1<f_{\chi}<200$, $3 \leq p<100,000$ and even integers $k$ with $2 \leq k \leq p-3$, there are $13,631,032,822$ pairs of $\left(p, \chi \omega^{k}\right)$ satisfying $(3-1)$. Among them, 288,086 pairs satisfy $\tilde{\lambda}_{p}\left(\chi \omega^{k}\right)=1,53$ pairs 


\begin{tabular}{c|l}
\hline$\nu_{p}(\chi)$ & \multicolumn{1}{|c}{$\left(f_{\chi}, p\right)$} \\
\hline 1 & $(8,31)(24,523)(33,29)(33,37)(37,7)(40,191)$ \\
& $(40,643)(41,7211)(57,59)(57,28927)(60,181)$ \\
& $(65,8831)(69,5)(73,41)(76,79)(85,3)(92,7)$ \\
& $(97,3331)(104,2683)(109,3)(109,5)(109,809)$ \\
& $(113,53)(113,20219)(124,157)(129,5419)$ \\
& $(136,37)(136,547)(136,4733)(140,23)$ \\
& $(140,577)(145,17)(145,37)(149,7)(156,5)$ \\
& $(156,7)(157,9613)(161,5)(165,199)(172,3)$ \\
& $(173,227)(181,3)(185,139)(185,2389)$ \\
\hline 2 & $(89,5)(69,17)$ \\
\hline
\end{tabular}

TABLE 1. The $\nu$-invariants of real quadratic fields.

$\tilde{\lambda}_{p}\left(\chi \omega^{k}\right)=2$, and two pairs $\tilde{\lambda}_{p}\left(\chi \omega^{k}\right)=3$. By the method of [Ichimura and Sumida 96], we verified Greenberg's conjecture, i.e., $\lambda_{p}\left(\chi \omega^{k}\right)=0$ for each of them. Moreover, we checked that $\nu_{p}\left(\chi \omega^{k}\right) \leq 2$. In the above range, 38 pairs do not satisfy $(3-1)$. For these cases, we checked that $\tilde{\lambda}_{p}\left(\chi \omega^{k}\right)=0$ if $\chi \omega^{k}(p)=1$, and that $\tilde{\lambda}_{p}\left(\chi \omega^{k}\right)=1$ if $\chi \omega^{1-k}(p)=1$, which implies that $\nu_{p}\left(\chi \omega^{k}\right)=0$. Further, by computation of the $p$-units of real quadratic fields $\mathbb{Q}\left(\sqrt{f_{\chi}}\right)$, we verified that $\lambda_{p}(\chi)=\lambda_{p}^{\prime}(\chi)=\nu_{p}^{\prime}(\chi)=0$ for all $f_{\chi}$ and $p$ in the above range. All pairs $\left(f_{\chi}, p\right)$ with $\nu_{p}(\chi)>0$ are given in Table 1 (see [Fukuda and Komatsu 86] and [Fukuda and Taya 95]).

Proposition 3.1. $\lambda_{p}\left(\mathbb{Q}\left(\sqrt{f_{\chi}}, \zeta_{p}+\zeta_{p}^{-1}\right)\right)=0$ for all $1<$ $f_{\chi}<200$ and $3 \leq p<100,000$.

Let us call a pair of integers $(p, k)$ a $\chi$-irregular pair if $p$ is a prime, $k$ is an even integer satisfying $2 \leq k \leq p-3, p$ divides $a_{0}\left(\chi \omega^{k}\right)=L_{p}\left(1, \chi \omega^{k}\right)\left(\right.$ or $\left.b_{0}\left(\chi \omega^{k}\right)=L_{p}\left(0, \chi \omega^{k}\right)\right)$, and $\left(p, \chi \omega^{k}\right)$ satisfies $(3-1)$. Further, we define the $\chi$ irregularity index $r_{p}(\chi)$ by

$$
r_{p}(\chi)=\sharp\{(p, k) \mid(p, k) \text { is a } \chi \text {-irregular pair }\} .
$$

We call a prime number $p \chi$-irregular if $r_{p}(\chi)>0$. Let $m_{p}(\chi)$ be the number of even integers $k$ with $2 \leq k \leq p-3$ such that $\left(p, \chi \omega^{k}\right)$ satisfies $(3-1)$. We define

$$
n_{r}=\sum_{(\chi, p) \text { s.t. } r_{p}(\chi)=r} 1
$$

and

$$
n_{r}^{\prime}=\sum_{\chi, p} m_{p}(\chi) C_{r}\left(\frac{1}{p}\right)^{r}\left(\frac{p-1}{p}\right)^{m_{p}(\chi)-r},
$$

where $\chi$ runs over all even quadratic characters with $1<f_{\chi}<200$, and $p$ runs over all prime numbers with $3 \leq p<100,000$. The distribution of the indices of $\chi$ irregularity is given in Table 2. The actual numbers $n_{r}$

\begin{tabular}{c|cc|cc}
\hline$r$ & $n_{r}$ & $n_{r}^{\prime}$ & the density & the density' \\
\hline 0 & 348574 & 349090.14 & 0.60579423 & 0.60669125 \\
1 & 174919 & 174464.73 & 0.30399548 & 0.30320601 \\
2 & 43596 & 43583.01 & 0.07576642 & 0.07574384 \\
3 & 7293 & 7257.27 & 0.01267466 & 0.01261257 \\
4 & 942 & 906.2 & 0.00163712 & 0.00157492 \\
5 & 73 & 90.51 & 0.00012686 & 0.00015730 \\
6 & 3 & 7.53 & 0.00000521 & 0.00001309 \\
7 & 0 & 0.53 & 0.00000000 & 0.00000093 \\
\hline
\end{tabular}

TABLE 2. The $\chi$-irregularity index density.

seem to be near the expected numbers $n_{r}^{\prime}$ (see [Washington 97 , page 63$]$ ).

In Tables 3-6, we extended the tables of [SumidaTakahashi 04] to all primes below 100, 000 .

In Figures 1-2, we compare the actual number of exceptional pairs with the expected number in the range $200<p<100,000$.

On the other hand, we found the following fact:

Proposition 3.2. For $f_{\chi}=5$ and $p<2,000,000$, there is no exceptional pair, that is, for any pair $\left(p, \chi \omega^{k}\right)$ which satisfies (3-1),

$$
\begin{aligned}
\nu_{p}\left(\chi \omega^{k}\right) & =0, \\
v_{p}\left(a_{0}\left(\chi \omega^{k}\right)\right) & =v_{p}\left(b_{0}\left(\chi \omega^{k}\right)\right) \\
& =\tilde{\lambda}_{p}\left(\chi \omega^{k}\right) \\
& \leq 1 .
\end{aligned}
$$

From our data, the actual number seems to be near the expected number. Even for large $p$, it might be possible that the actual number is near the expected number. Therefore, it is not very strange that we have not been able to find any exceptional pair for $\chi=\chi^{0}$, especially any counterexample to Vandiver's conjecture.

\section{HIGHER $K$-GROUPS OF THE RING OF INTEGERS OF $\mathbb{Q}\left(\sqrt{f_{\chi}}\right)$}

In order to compute the orders of the étale cohomology groups, we prepare some notation. For an odd integer $m$, we write the Iwasawa polynomial $g_{\chi \omega^{1-m}}(T)$ for the $p$-adic $L$-function $L_{p}\left(s, \chi \omega^{1-m}\right)$ in the form

$$
g_{\chi \omega^{1-m}}(T)=\prod_{i=1}^{\tilde{\lambda}\left(\chi \omega^{1-m}\right)}\left(T-\alpha_{\chi \omega^{1-m}, i}\right), \quad \alpha_{\chi \omega^{1-m}, i} \in \overline{\mathbb{Q}}_{p} .
$$

We let

$$
x(p, \chi, m-1)=\min \{A, B\} .
$$




\begin{tabular}{ccc|ccc|ccc|ccc}
\hline$f_{\chi}$ & $p$ & $k$ & $f_{\chi}$ & $p$ & $k$ & $f_{\chi}$ & $p$ & $k$ & $f_{\chi}$ & $p$ & $k$ \\
\hline 8 & 34301 & 114 & 12 & 701 & 542 & 21 & 199 & 150 & 33 & 53 & 30 \\
37 & 43 & 32 & 53 & 1033 & 564 & 56 & 55621 & 9294 & 69 & 19 & 14 \\
85 & 3697 & 3086 & 88 & 71 & 26 & 101 & 5333 & 2770 & 104 & 19 & 14 \\
113 & 43 & 32 & 113 & 3373 & 1602 & 124 & 197 & 126 & 124 & 239 & 48 \\
129 & 67 & 28 & 140 & 4751 & 120 & 141 & 5431 & 4826 & 149 & 43 & 32 \\
149 & 71 & 16 & 149 & 229 & 182 & 156 & 50051 & 4582 & 157 & 401 & 56 \\
161 & 101 & 22 & 168 & 37 & 22 & 172 & 73 & 10 & 173 & 7 & 4 \\
173 & 43 & 32 & 173 & 101 & 42 & 177 & 17 & $6 *$ & 181 & 71 & 52 \\
181 & 6991 & 1628 & 185 & 827 & 354 & 188 & 1621 & 168 & 193 & 62791 & 57100 \\
197 & 521 & 372 & & & & & & & & & \\
\hline
\end{tabular}

TABLE 3. $\nu_{p}\left(\chi \omega^{k}\right)=1$ (2 for the $*$-marked case $)$.

\begin{tabular}{ccc|ccc|ccc|ccc}
\hline$f_{\chi}$ & $p$ & $k$ & $f_{\chi}$ & $p$ & $k$ & $f_{\chi}$ & $p$ & $k$ & $f_{\chi}$ & $p$ & $k$ \\
\hline 8 & 59 & 36 & 17 & 61 & 32 & 21 & 149 & 128 & 21 & 10169 & 7388 \\
28 & 977 & 828 & 33 & 59 & 42 & 37 & 1091 & 812 & 40 & 12101 & 318 \\
41 & 7 & 4 & 41 & 283 & 102 & 44 & 787 & 148 & 53 & 7 & 2 \\
53 & 1879 & 1158 & 57 & 2161 & 758 & 61 & 17 & 4 & 61 & 1747 & 1270 \\
76 & 191 & 84 & 88 & 35099 & 24446 & 89 & 41 & 10 & 92 & 181 & 124 \\
97 & 17 & 4 & 105 & 769 & 524 & 105 & 1453 & 162 & 120 & 2749 & 2196 \\
124 & 41 & 30 & 124 & 26227 & 13770 & 140 & 107 & 74 & 149 & 797 & 140 \\
149 & 2767 & 2178 & 152 & 17 & 12 & 152 & 25453 & 15704 & 156 & 66877 & 48258 \\
168 & 43 & 10 & 173 & 13 & 4 & 177 & 31 & 24 & 184 & 373 & 72 \\
193 & 7873 & 1886 & & & & & & & & & \\
\hline
\end{tabular}

TABLE 4. $v_{p}\left(a_{0}\left(\chi \omega^{k}\right)\right)=2$.

\begin{tabular}{ccc|ccc|ccc|ccc}
\hline$f_{\chi}$ & $p$ & $k$ & $f_{\chi}$ & $p$ & $k$ & $f_{\chi}$ & $p$ & $k$ & $f_{\chi}$ & $p$ & $k$ \\
\hline 8 & 2221 & 1600 & 13 & 109 & 6 & 17 & 1319 & 88 & 28 & 223 & 126 \\
33 & 31 & 24 & 33 & 1777 & 1184 & 41 & 19 & 12 & 41 & 421 & 126 \\
60 & 19 & 14 & 61 & 7481 & 3516 & 73 & 11 & 2 & 73 & 1487 & 808 \\
76 & 1451 & 418 & 76 & 4283 & 3484 & 89 & 23369 & 9986 & 97 & 367 & 26 \\
97 & 13613 & 13022 & 109 & 41 & 32 & 133 & 1061 & 446 & 136 & 449 & 284 \\
152 & 41 & 2 & 152 & 4027 & 3108 & 156 & 4637 & 2280 & 156 & 38891 & 9454 \\
157 & 8221 & 582 & 165 & 29 & 26 & 165 & 89 & 66 & 165 & 1229 & 48 \\
172 & 11 & 4 & 172 & 1487 & 900 & 177 & 337 & 74 & 177 & 58787 & 20838 \\
184 & 1171 & 464 & 185 & 167 & 68 & 188 & 89 & 76 & & & \\
\hline
\end{tabular}

TABLE 5. $v_{p}\left(b_{0}\left(\chi \omega^{k}\right)\right)=2$.

\begin{tabular}{ccc|ccc|ccc|ccc}
\hline$f_{\chi}$ & $p$ & $k$ & $f_{\chi}$ & $p$ & $k$ & $f_{\chi}$ & $p$ & $k$ & $f_{\chi}$ & $p$ & $k$ \\
\hline 8 & 1151 & 842 & 8 & 27791 & 11840 & 21 & 11 & 4 & 21 & 60637 & 16528 \\
24 & 29 & 4 & 24 & 181 & 84 & 29 & 569 & 64 & 37 & 5 & 2 \\
37 & 89 & 66 & 37 & 3251 & 1094 & 40 & 257 & 232 & 44 & 653 & 448 \\
53 & 193 & 14 & 56 & 1663 & 616 & 60 & 1277 & 582 & 60 & 1481 & 986 \\
65 & 18121 & 3044 & 92 & 5 & 2 & 97 & 271 & 94 & 104 & 19 & 14 \\
104 & 7919 & 4386 & 105 & 373 & 340 & 109 & 131 & 100 & 109 & 293 & 132 \\
109 & 373 & 128 & 124 & 733 & 58 & 124 & 2111 & 1480 & 124 & 22091 & 15370 \\
129 & 23 & 4 & 133 & 911 & 196 & 136 & 71 & 20 & 137 & 17 & 8 \\
140 & 23 & 10 & 140 & 367 & 292 & 141 & 113 & 108 & 141 & 5939 & 2938 \\
145 & 43 & 28 & 145 & 61 & 58 & 145 & 167 & 128 & 145 & 4157 & 3528 \\
149 & 5 & 2 & 149 & 509 & 426 & 161 & 2389 & 646 & 161 & 64879 & 57186 \\
165 & 11 & 2 & 165 & 23 & $6 *$ & 165 & 71089 & 24840 & 172 & 13 & 10 \\
172 & 47 & 38 & 173 & 7 & 4 & 177 & 157 & 48 & 181 & 223 & 26 \\
181 & 82007 & 51630 & 185 & 17 & $10 *$ & 185 & 17 & 6 & & & \\
\hline
\end{tabular}

TABLE 6. $\tilde{\lambda}\left(\chi \omega^{k}\right)=2$ (3 for the $*$-marked cases $)$. 


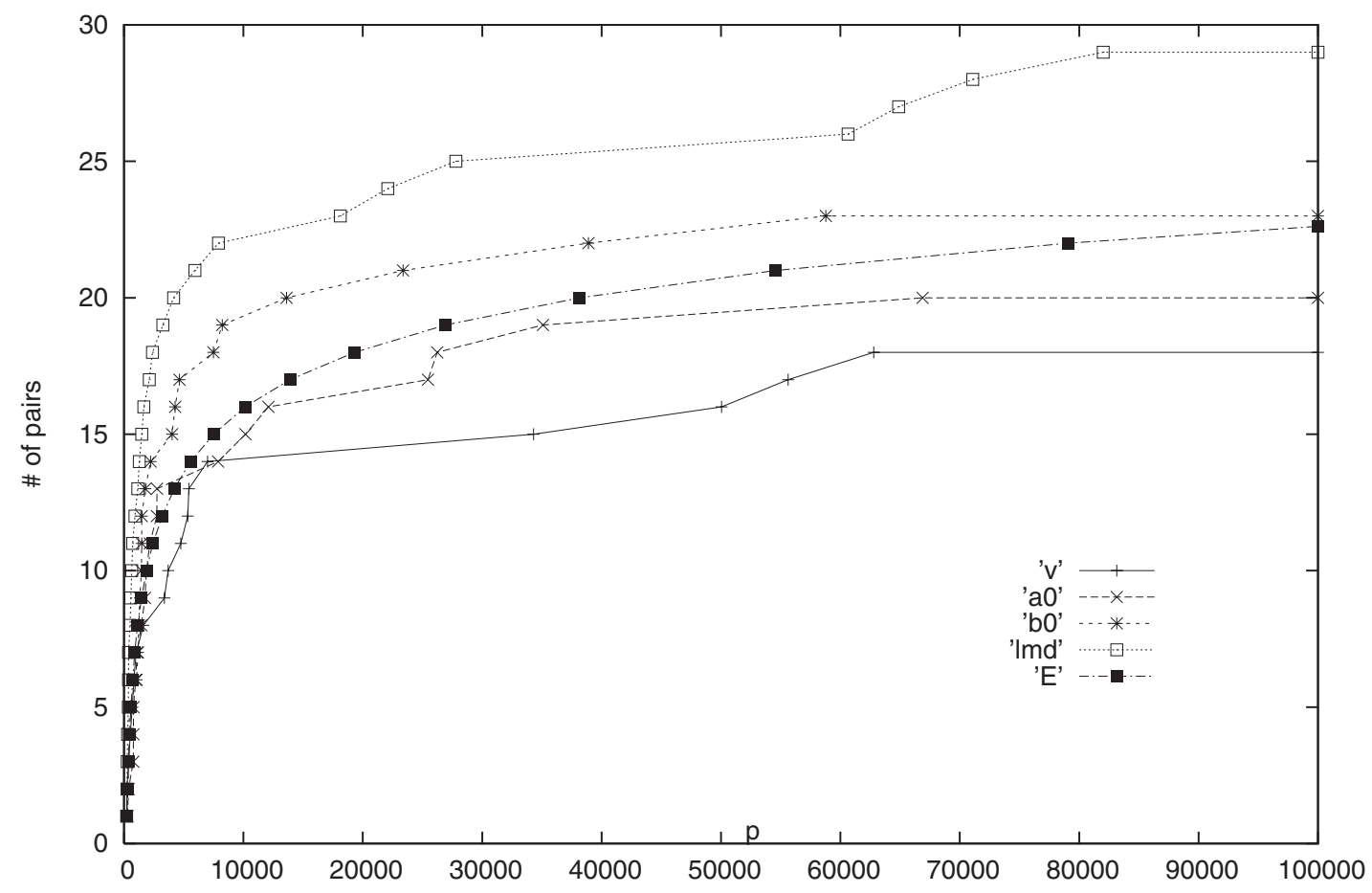

FIGURE 1. Exceptional pairs $(200<p<100,000)$.

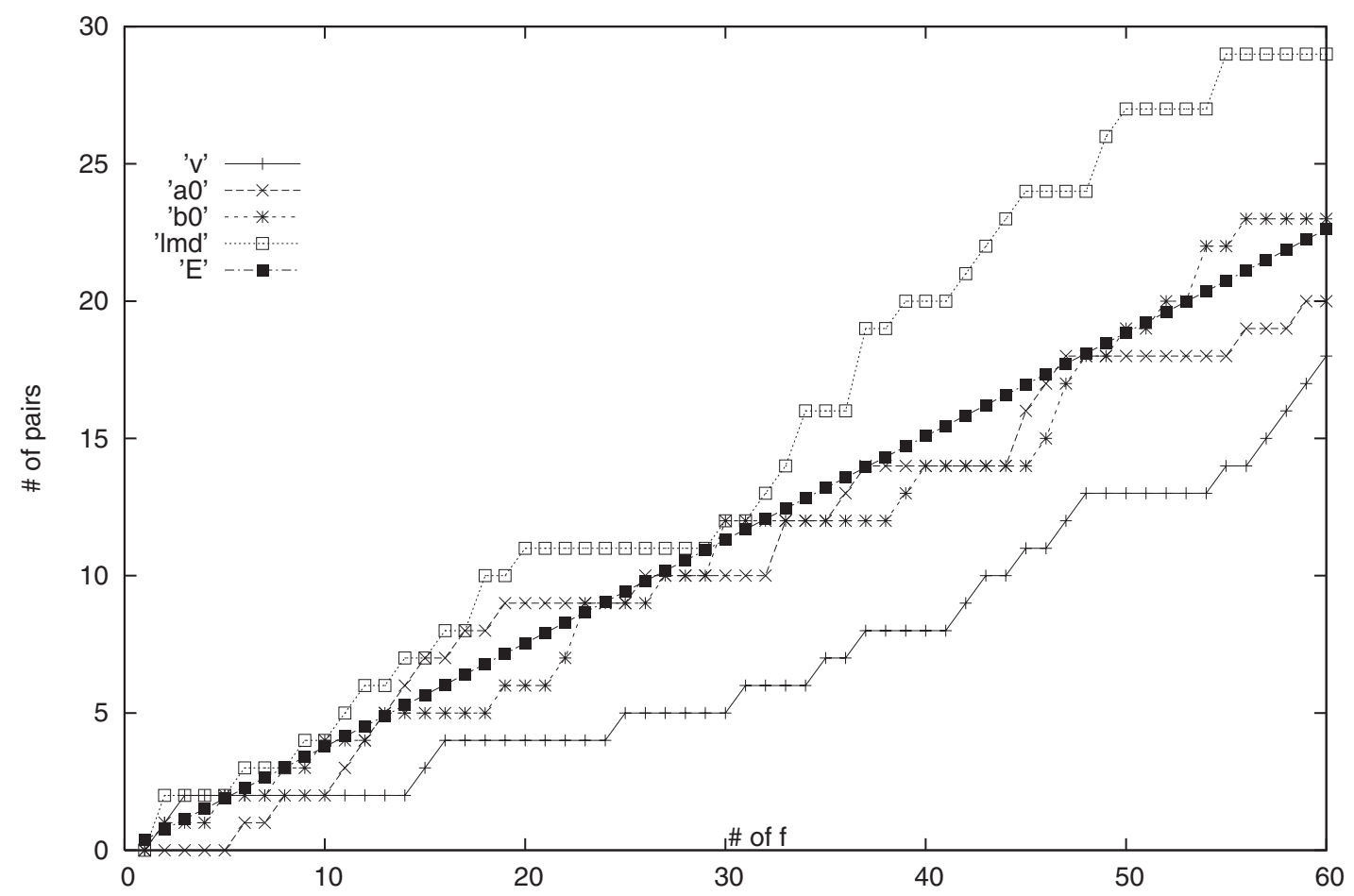

FIGURE 2. Exceptional pairs $(200<p<100,000)$. 
where

$$
\begin{aligned}
& A=\nu_{p}\left(\chi \omega^{1-m}\right) \\
& B=v_{p}\left(\prod_{i=1}^{\tilde{\lambda}\left(\chi \omega^{1-m}\right)}\left(1-\left(1+f_{0}\right)^{m-1}\left(\alpha_{\chi \omega^{1-m}, i}+1\right)\right)\right)
\end{aligned}
$$

For an even integer $m$, let $\alpha_{\chi \omega^{m}, i}^{*}=\frac{f_{0}-\alpha_{\chi \omega} m, i}{1+\alpha_{\chi \omega^{m}, i}}$, $g_{\chi \omega^{m}}^{*}(T)=\prod_{i=1}^{\tilde{\lambda}\left(\chi \omega^{m}\right)}\left(T-\alpha_{\chi \omega^{m}, i}^{*}\right)$, and

$$
\begin{aligned}
& x^{*}(p, \chi, m-1)= \\
& v_{p}\left(\prod_{i=1}^{\tilde{\lambda}\left(\chi \omega^{m}\right)}\left(1-\left(1+f_{0}\right)^{m-1}\left(\alpha_{\chi \omega^{m}, i}^{*}+1\right)\right)\right) .
\end{aligned}
$$

Further, for an integer $m$, we define the following sets of prime numbers

$$
\begin{aligned}
& S_{1}(\chi, m-1)=\left\{\begin{array}{c}
p: \frac{p-1}{2} \mid(m-1), \\
(p-1) \nmid(m-1), \\
\chi \omega^{\frac{p-1}{2}}(p)=1, \\
\chi \omega^{\frac{p-1}{2}} \neq \chi^{0}
\end{array}\right\}, \\
& S_{2}(\chi, m-1)=\{p:(p-1) \mid(m-1) \text { and } \chi(p)=1\} .
\end{aligned}
$$

We set

$$
y(p, \chi, m-1)=\left\{\begin{array}{lc}
v_{p}(m-1)+1 & \text { if } p \in S_{1}(\chi, m-1) \\
0 & \cup S_{2}(\chi, m-1) \\
& \text { otherwise }
\end{array}\right.
$$

Proposition 4.1. Let $\chi$ be an even quadratic Dirichlet character, $p$ an odd prime number, and $F=F_{\chi}$. For an even integer $m$, if $\left(p, \chi \omega^{m}\right)$ satisfies $(3-1)$, then

$$
\sharp X_{\infty}^{\prime}(m-1)_{G_{\infty}}^{\chi}=p^{x^{*}(p, \chi, m-1)} .
$$

For an odd integer $m$, assume that $X_{\infty}^{\prime \chi \omega^{1-m}}$ is finite. If $\left(p, \chi \omega^{1-m}\right)$ satisfies $(3-1)$ and if $g_{\chi \omega^{1-m}}(T)$ is an Eisenstein polynomial or of degree one, then

$$
\sharp X_{\infty}^{\prime}(m-1)_{G_{\infty}}^{\chi}=p^{x(p, \chi, m-1)} .
$$

Further, for an integer $m$, we have

$$
\frac{\sharp \prod_{v \mid p} H^{2}\left(F_{v}, \mathbb{Z}_{p}(m)\right)^{\chi}}{\sharp H^{0}\left(\mathcal{O}_{F}, \mathbb{Q}_{p} / \mathbb{Z}_{p}(1-m)\right)^{\chi}}=p^{y(p, \chi, m-1)} .
$$

Proof: We first set some notation. Let $\gamma$ be the topological generator of $\Gamma$ such that $\zeta_{f_{0} p^{n}}^{\gamma}=\zeta_{f_{0} p^{n}}^{1+f_{0}}$ for all $n \geq 0$. As usual, we can identify the completed group ring $\mathbb{Z}_{p}[[\Gamma]]$ with the formal power series ring $\Lambda=\mathbb{Z}_{p}[[T]]$ by $\gamma=1+T$. For a finitely generated torsion $\Lambda$-module $A$, we define the Iwasawa polynomial $\operatorname{char}_{\Lambda}(A)$ to be the characteristic polynomial of the action $T$ on $A \otimes \mathbb{Q}_{p}$ (see [Washington 97, Section 13]). By (3-1) and [Mazur and Wiles 84], $\operatorname{char}_{\Lambda}\left(X^{\prime \chi \omega^{1-m}}\right)=g_{\chi \omega^{m}}^{*}(T)$. Since

$$
X_{\infty}^{\prime}(m-1)_{\Delta}^{\chi} \simeq X_{\infty}^{\prime \chi \omega^{1-m}} \otimes \mathbb{Z}_{p}(m-1),
$$

we have

$$
\begin{aligned}
\operatorname{char}_{\Lambda}\left(X^{\prime}(m-1)_{\Delta}^{\chi}\right) & = \\
\tilde{\lambda}\left(\chi \omega^{m}\right) & \\
& \prod_{i=1}\left(T+1-\left(1+f_{0}\right)^{m-1}\left(\alpha_{\chi \omega^{m}, i}^{*}+1\right)\right) .
\end{aligned}
$$

Since $X^{\prime}{ }_{\infty}(m-1)_{\Delta}^{\chi}$ has no nontrivial finite $\Lambda$-submodule, the order of the $\Gamma$-coinvariant quotient is obtained from the constant term of the characteristic polynomial: $v_{p}\left(\sharp A / A^{\gamma-1}\right)=v_{p}(\sharp A / T A)=v_{p}\left(\left.\operatorname{char}_{\Lambda}(A)\right|_{T=0}\right)$. Hence, we obtain Equation (4-1).

Let $M_{\infty}$ be the maximal abelian $p$-extension of $K_{\infty}$ unramified outside $p$. Set $Y_{\infty}=\operatorname{Gal}\left(M_{\infty} / K_{\infty}\right)$ and $D_{\infty}=\operatorname{Gal}\left(M_{\infty} / L_{\infty}^{\prime}\right)$. By definition, $X_{\infty}^{\prime}=$ $Y_{\infty} / D_{\infty}$. By $(3-1)$ and [Mazur and Wiles 84], we have $\operatorname{char}_{\Lambda}\left(Y_{\infty}^{\chi \omega^{1-m}}\right)=g_{\chi \omega^{1-m}}(T)$. Hence,

$$
\begin{aligned}
& \operatorname{char}_{\Lambda}\left(Y_{\infty}(m-1)_{\Delta}^{\chi}\right)= \\
& \quad \prod_{i=1}^{\tilde{\lambda}\left(\chi \omega^{1-m}\right)}\left(T+1-\left(1+f_{0}\right)^{m-1}\left(\alpha_{\chi \omega^{1-m}, i}+1\right)\right) .
\end{aligned}
$$

Since $Y_{\infty}(m-1)_{\Delta}^{\chi}$ has no nontrivial finite $\Lambda$-submodule, by the assumption on $g_{\chi \omega^{1-m}}(T)$, we can completely distinguish any $\Lambda$-submodules of $Y_{\infty}(m-1)_{\Delta}^{\chi}$ by their indices. Hence, $D_{\infty}^{\chi \omega^{1-m}}$ is the submodule of $Y_{\infty}^{\chi \omega^{1-m}}$ of index $p^{\nu_{p}\left(\chi \omega^{1-m}\right)}$. Therefore, Equation (4-2) follows.

Set $z=\sqrt{(-1)^{\frac{p-1}{2}} p}$. Then, $\mathbb{Q}(z)$ (respectively $\mathbb{Q}(z \sqrt{f}))$ is associated to $\eta=\omega^{\frac{p-1}{2}}$ (respectively $\chi \eta$ ). In order to prove Equation (4-3), we first calculate $h_{2, v}=\sharp H^{2}\left(F_{v}, \mathbb{Z}_{p}(m)\right)$. By local duality, we have $h_{2, v}=$ $\sharp H^{0}\left(F_{v}, \mathbb{Q}_{p} / \mathbb{Z}_{p}(1-m)\right)=\sharp H^{0}\left(F_{v}, \mathbb{Q}_{p} / \mathbb{Z}_{p}(m-1)\right)$. If $z \notin F_{v}$, that is, $\chi \eta(p) \neq 1$, we have

$$
h_{2, v}= \begin{cases}p^{v_{p}(m-1)+1} & \text { if }(p-1) \mid(m-1), \\ 1 & \text { otherwise }\end{cases}
$$

If $z \in F_{v}$, that is, $\chi \eta(p)=1$, we have

$$
h_{2, v}= \begin{cases}p^{v_{p}(m-1)+1} & \text { if } \frac{p-1}{2} \mid(m-1), \\ 1 & \text { otherwise. }\end{cases}
$$


Similarly, we can calculate $h_{0}=\sharp H^{0}\left(\mathcal{O}_{F}, \mathbb{Q}_{p} / \mathbb{Z}_{p}(1-\right.$ $m))=\sharp H^{0}\left(\mathcal{O}_{F}, \mathbb{Q}_{p} / \mathbb{Z}_{p}(m-1)\right)$. If $z \notin F$, that is, $\chi \eta \neq \chi^{0}$, we have

$$
h_{0}= \begin{cases}p^{v_{p}(m-1)+1} & \text { if }(p-1) \mid(m-1), \\ 1 & \text { otherwise }\end{cases}
$$

If $z \in F$, that is, $\chi \eta=\chi^{0}$, we have

$$
h_{0}= \begin{cases}p^{v_{p}(m-1)+1} & \text { if } \frac{p-1}{2} \mid(m-1) \\ 1 & \text { otherwise }\end{cases}
$$

(I) If $\chi \eta=\chi^{0}$, then $\chi(p)=0 \neq 1$ and $\chi \eta(p)=1$. Hence, we have $h_{2, v}=h_{0}$.

(II) If $\chi \eta \neq \chi^{0}$ and $\chi(p)=1$, then $\chi \eta(p)=0 \neq 1$. Hence, we have $h_{2, v_{1}} h_{2, v_{2}}=1^{2}$ and $h_{0}=1$ if $(p-1) \nmid(m-1)$. If $(p-1) \mid(m-1)$, we have $h_{2, v_{1}} h_{2, v_{2}}=\left(p^{v_{p}(m-1)+1}\right)^{2}$ and $h_{0}=p^{v_{p}(m-1)+1}$. Since $\chi(p)=1$ implies $\chi \eta \neq \chi^{0}$, such a prime number $p$ is included in $S_{2}(\chi, m-1)$.

(III) If $\chi \eta \neq \chi^{0}, \chi(p) \neq 1$, and $\chi \eta(p) \neq 1$, then we have $h_{2, v}=h_{0}$.

(IV) If $\chi \eta \neq \chi^{0}, \chi(p) \neq 1$, and $\chi \eta(p)=1$, then we have $h_{2, v}=h_{0}$ unless $\frac{p-1}{2} \mid(m-1)$ and $(p-1) \nmid(m-1)$. If $\frac{p-1}{2} \mid(m-1)$ and $(p-1) \nmid(m-1)$, we have $h_{2, v}=$ $p^{v_{p}(m-1)+1}$ and $h_{0}=1$. Since $\chi \eta(p)=1$ implies $\chi(p)=$ $0 \neq 1$, such a prime number $p$ is included in $S_{1}(\chi, m-1)$. Hence, we obtain Equation (4-3).

For a positive integer $m$ and a prime number $p$, we denote by $K_{2 m-2}\left(\mathcal{O}_{F}\right)(p)$ the $p$-Sylow subgroup of $K_{2 m-2}\left(\mathcal{O}_{F}\right)$. Here we set

$$
\begin{gathered}
K_{2 m-2}^{\prime}\left(\mathcal{O}_{F}\right)=\bigoplus_{3 \leq p<100,000} K_{2 m-2}\left(\mathcal{O}_{F}\right)(p), \\
X^{\prime}(\chi, m-1)=\prod_{3 \leq p<100,000} \sharp X_{\infty}^{\prime}(m-1)_{G_{\infty}}^{\chi}, \text { and } \\
Y_{i}^{\prime}(\chi, m-1)= \\
\prod_{p \in S_{i}(\chi, m), 3 \leq p<100,000} \frac{\sharp \prod_{v \mid p} H^{2}\left(F_{v}, \mathbb{Z}_{p}(m)\right)^{\chi}}{\left.\sharp \mathcal{O}_{F}, \mathbb{Q}_{p} / \mathbb{Z}_{p}(1-m)\right)^{\chi}} .
\end{gathered}
$$

Then, by Theorem 2.4 and the surjectivity of $p$-adic Chern characters, we have

$$
\sharp K_{2 m-2}^{\prime}\left(\mathcal{O}_{F}\right)^{\chi}
$$

is divided by

$$
X^{\prime}(\chi, m-1) \cdot Y_{1}^{\prime}(\chi, m-1) \cdot Y_{2}^{\prime}(\chi, m-1) .
$$

For an even integer $m$ and a prime number $p$ which divides the numerator of $B_{m, \chi}$, we can compute
$v_{p}\left(X^{\prime}(\chi, m-1)\right)$ from the zeros of the Iwasawa polynomial by Proposition 4.1. In fact, we can easily obtain a lot of examples of $(\chi, m)$ with $X^{\prime}(\chi, m-1)>1$. On the other hand, for an odd integer $m$, it is more difficult to obtain examples of $(\chi, m)$ with $X^{\prime}(\chi, m-1)>1$. Since Vandiver's conjecture is true for all $p<12,000,000$, $X_{\infty}^{\prime}(m-1)_{G_{\infty}}^{\chi^{0}}$ is trivial for any odd integer $m$. Further, we have $\sharp H^{2}\left(\mathbb{Q}_{p}, \mathbb{Z}_{p}(m)\right)=\sharp H^{0}\left(\mathbb{Q}_{p}, \mathbb{Q}_{p} / \mathbb{Z}_{p}(1-m)\right)=$ $\sharp H^{0}\left(\mathbb{Z}, \mathbb{Q}_{p} / \mathbb{Z}_{p}(1-m)\right)$. By Theorem 2.4, Proposition 4.1, and our computational result, we obtain such examples in Table 7.

We have $Y_{1}^{\prime}\left(\chi, 2 m^{\prime}\right)=1$ for all the cases in Table 7 . If the Quillen-Lichtenbaum conjecture is true, there is no other factor of $\sharp K_{4 m^{\prime}}^{\prime}\left(\mathcal{O}_{\mathbb{Q}\left(\sqrt{f_{\chi}}\right)}\right)$ : for example,

$$
K_{96}\left(\mathcal{O}_{\mathbb{Q}(\sqrt{21})}\right) \supseteq K_{96}^{\prime}\left(\mathcal{O}_{\mathbb{Q}(\sqrt{21})}\right) \simeq \mathbb{Z} /(5 \cdot 17 \cdot 199 \mathbb{Z}) .
$$

In [Soulé 03], an explicit huge bound is given for the order of $K_{4 m^{\prime}}\left(\mathcal{O}_{F}\right)$. However, it would be impossible to compute $x\left(p, \chi, 2 m^{\prime}\right)$ by our method up to the bound. Therefore it is possible that $\sharp K_{4 m^{\prime}}\left(\mathcal{O}_{F}\right)$ is divisible by a prime number larger than 100,000.

\section{ALGORITHMS FOR COMPUTING ARITHMETIC ELEMENTS}

In order to make the tables in the previous sections, we computed the following arithmetic elements:

(I) the generalized Bernoulli numbers modulo $p$, i.e., $\sum_{k=0}^{p-3} B_{k, \chi} t^{k} / k ! \bmod p$,

$(\mathrm{II})_{n}$ the Iwasawa polynomial $g_{\chi \omega^{k}}(T) \bmod p^{n+1}$,

$(\mathrm{III})_{n}$ the special cyclotomic unit $c_{n}^{Y_{n}(T)}$ modulo a prime ideal $\mathfrak{L}_{n}$, and

$(\mathrm{IV})_{n}$ the Gauss sum $g_{0}\left(\mathfrak{L}_{0}\right)$ modulo a prime ideal $\mathfrak{L}_{0}^{*}$, where $\mathfrak{L}_{0}=N_{K_{n} / K_{0}} \mathfrak{L}_{n}$.

By (I), we obtain $\chi$-irregular pairs $(p, k)$. By $(\mathrm{II})_{n}$, we obtain information on the $p$-adic $L$-function $L_{p}\left(s, \chi \omega^{k}\right)$. By Mazur-Wiles' theorem, if $\left(p, \chi \omega^{k}\right)$ satisfies $(3-1)$, $\sharp A_{n}^{\prime} \chi \omega^{k}=\sharp\left(E_{n} / C_{n}\right)(p)^{\chi \omega^{k}}$, where $E_{n}$ is the group of units of $K_{n}$, and $C_{n}$ is the group of cyclotomic units of $K_{n}$. Since $X_{\infty}^{\prime}{ }^{\chi \omega^{k}}=\lim _{\leftarrow} A_{n}^{\prime}{ }^{\chi \omega^{k}}$, we obtain information on the Iwasawa invariants $\lambda_{p}\left(\chi \omega^{k}\right)$ and $\nu_{p}\left(\chi \omega^{k}\right)$ from $\sharp\left(E_{n} / C_{n}\right)(p)^{\chi \omega^{k}}$. By [Sumida-Takahashi 04, Theorem 1], using (III $)_{n}$ and (IV) $)_{n}$, we can compute the order of $\sharp\left(E_{n} / C_{n}\right)(p)^{\chi \omega^{k}}$ without directly computing $E_{n}$ (see [Schoof 03]).

Here we briefly explain some effective algorithms for computing the above elements. For simplicity, we assume that $p$ does not divide $f=f_{\chi}$. 


\begin{tabular}{c|c|c|l||c|c|c|l}
\hline $4 m^{\prime}$ & $f_{\chi}$ & $X^{\prime}\left(\chi, 2 m^{\prime}\right)$ & $Y_{2}^{\prime}\left(\chi, 2 m^{\prime}\right)$ & $4 m^{\prime}$ & $f_{\chi}$ & $X^{\prime}\left(\chi, 2 m^{\prime}\right)$ & $Y_{2}^{\prime}\left(\chi, 2 m^{\prime}\right)$ \\
\hline 68372 & 8 & 34301 & 1 & 316 & 12 & 701 & 1 \\
96 & 21 & 199 & $5 \cdot 17$ & 44 & 33 & 53 & 1 \\
20 & 37 & 43 & $3 \cdot 11$ & 936 & 53 & 1033 & $7 \cdot 13^{2} \cdot 37$ \\
92652 & 56 & 55621 & $43 \cdot 6619 \cdot 15443$ & 8 & 69 & 19 & 5 \\
1220 & 85 & 3697 & 3 & 88 & 88 & 71 & 3 \\
5124 & 101 & 5333 & $43 \cdot 367$ & 8 & 104 & 19 & 5 \\
20 & 113 & 43 & 11 & 3540 & 113 & 3373 & $7 \cdot 11 \cdot 31$ \\
140 & 124 & 197 & $3 \cdot 11$ & 380 & 124 & 239 & $3 \cdot 11$ \\
76 & 129 & 67 & 1 & 9260 & 140 & 4751 & 1 \\
1208 & 141 & 5431 & 5 & 20 & 149 & 43 & 1 \\
108 & 149 & 71 & $7 \cdot 19$ & 92 & 149 & 229 & 47 \\
90936 & 156 & 50051 & $5 \cdot 7 \cdot 19$ & 688 & 157 & 401 & $3 \cdot 173$ \\
156 & 161 & 101 & 1 & 28 & 168 & 37 & 1 \\
124 & 172 & 73 & 3 & 4 & 173 & 7 & 1 \\
20 & 173 & 43 & 1 & 116 & 173 & 101 & 1 \\
20 & 177 & 17 & 11 & 36 & 181 & 71 & $3^{3}$ \\
10724 & 181 & 6991 & 3 & 944 & 185 & 827 & 1 \\
2904 & 188 & 1621 & $23 \cdot 67 \cdot 727$ & 11380 & 193 & 62791 & 3 \\
296 & 197 & 521 & 1 & & & & \\
\hline
\end{tabular}

TABLE 7. Factors of $\sharp K_{4 m^{\prime}}^{\prime}\left(\mathcal{O}_{\mathbb{Q}\left(\sqrt{f_{\chi}}\right)}\right)$ with $X^{\prime}\left(\chi, 2 m^{\prime}\right)>1$.

(I) We first compute the inversion of power series $\left(e^{f t}\right.$ 1) $/ t$ modulo $\left(p, t^{p-2}\right)$ by the method of [Knuth 81 , Section 4.7], in which we use the Fast Fourier Transform (FFT) algorithm (see [Knuth 81, Section 4.3.3]). Next, we compute the approximated polynomial $\sum_{a=1}^{f} \chi(a) e^{a t}$ modulo $\left(p, t^{p-2}\right)$. Finally, we multiply the two polynomials by using the FFT algorithm again.

(II) ${ }_{n}$ By [Washington 97, Theorem 5.11], we have

$$
\begin{aligned}
-L_{p}\left(1, \chi \omega^{k}\right) & \equiv-L_{p}\left(1-k, \chi \omega^{k}\right) \\
& =\left(1-\chi \omega^{k} \omega^{-k}(p) p^{k-1}\right) \frac{B_{k, \chi \omega^{k} \omega^{-k}}}{k} \\
& =\left(1-\chi(p) p^{k-1}\right) \frac{B_{k, \chi}}{k} \\
& \equiv \frac{B_{k, \chi}}{k} \bmod p .
\end{aligned}
$$

Therefore, from the result of (I), we can obtain indices $k$ such that $p$ divides $L_{p}\left(1, \chi \omega^{k}\right)=g_{\chi \omega^{k}}(0) u_{\chi \omega^{k}}(0)$. In order to effectively compute $g_{\chi \omega^{k}}(T) \bmod p^{n+1}$, we use the following Theorem 5.1.

Theorem 5.1. [Washington 97, Section 5.2] We have the formula

$$
\begin{aligned}
L_{p}\left(s, \chi \omega^{k}\right)= & \frac{1}{f_{0}} \frac{1}{s-1} \sum_{a=1, p \nmid a}^{f_{0}} \chi \omega^{k}(a)\langle a\rangle^{1-s} \\
& \times \sum_{j=0}^{\infty}\left(\begin{array}{c}
1-s \\
j
\end{array}\right)\left(B_{j}\right)\left(\frac{f_{0}}{a}\right)^{j},
\end{aligned}
$$

where $\langle a\rangle=a \omega^{-1}(a)$. $(\text { III })_{n} \quad$ By using the Iwasawa polynomial $g_{\chi \omega^{k}}(T) \bmod p^{n+1}$, we define a polynomial $Y_{n}(T) \in \mathbb{Z}[T]$ (see [Ichimura and Sumida 96]). Then we can study the difference between the group of global units and that of cyclotomic units from the information on the special cyclotomic unit $c_{n}^{Y_{n}(T)} \bmod \mathfrak{L}_{n}$ for some prime ideals $\mathfrak{L}_{n}$ of $K_{n}$ of degree one. From this information we can obtain an upper bound for the order of the $p$-part of the ideal class group by Mazur-Wiles' theorem. For details, see [Sumida-Takahashi 04, Section 2].

$(\mathbf{I V})_{n}$ We can make certain that the computation (III) $)_{n}$ gives the exact value of the order by studying the Gauss sums $g_{0}\left(\mathfrak{L}_{0}\right) \bmod \mathfrak{L}_{0}^{*}$ for some prime ideals $\mathfrak{L}_{0}^{*}$ of $K_{0}$. In order to effectively compute $g_{0}\left(\mathfrak{L}_{0}\right) \bmod \mathfrak{L}_{0}^{*}$, we use the FFT algorithm once again. For details, see [SumidaTakahashi 04, Section 3].

Thirty personal computers were used for the computations in Section 3. The programs were written in UBASIC and C, in which the GNU MP library was included in order to multiply polynomials of large degree. For example, for $p=55,621$ and $f_{\chi}=56$, the calculations took about (I) 7 , (II) $)_{1} 30,(\mathrm{III})_{0} 7,(\mathrm{III})_{1} 7.4 \times 10^{5}$, and $(\mathrm{IV})_{0}$ $4.8 \times 10^{2}$ seconds on one PC (CPU: Pentium IV, 2.6-GHz, RAM: 1GB).

\section{ACKNOWLEDGMENTS}

The author wishes to express his gratitude to Professor H. Nakahara and the Division of Mathematical and Information Sciences, Faculty of Integrated Arts and Sciences, Hiroshima University for the help with the computations. He 
also wishes to express his gratitude to the referee for valuable comments. The author is partially supported by the Grantsin-Aid for Encouragement of Young Scientists (No.16740019) from Japan Society for the Promotion of Science.

\section{REFERENCES}

[Buhler et al. 93] J. Buhler, R. Crandall, R. Ernvall, and T. Metsänkylä. "Irregular Primes and Cyclotomic Invariants to Four Million." Math. Comp. 61 (1993), 151-153.

[Buhler et al. 01] J. Buhler, R. Crandall, R. Ernvall, T. Metsänkylä, and A. M. Shokrollahi. "Irregular Primes and Cyclotomic Invariants to 12 Million." J. Symbolic Comput. 31 (2001), 89-96.

[Dwyer and Friedlander 85] W. Dwyer and E. Friedlander. "Algebraic and Étale K-Theory." Trans. Amer. Math. Soc. 292 (1985), 247-280.

[Ferrero and Washington 79] B. Ferrero and L. Washington. "The Iwasawa Invariant $\mu_{p}$ Vanishes for Abelian Number Fields." Ann. of Math. 109 (1979), 377-395.

[Fukuda and Komatsu 86] T. Fukuda and K. Komatsu. "On $\mathbb{Z}_{p}$-Extensions of Real Quadratic Fields." J. Math. Soc. Japan 38 (1986), 95-102.

[Fukuda and Taya 95] T. Fukuda and H. Taya. "The Iwasawa $\lambda$-Invariants of $\mathbb{Z}_{p}$-Extensions of Real Quadratic Fields." Acta Arith. 69 (1995), 277-292.

[Ichimura and Sumida 96] H. Ichimura and H. Sumida. "On the Iwasawa Invariants of Certain Real Abelian Fields II." Internat. J. Math. 7 (1996), 721-744.

[Iwasawa 72] K. Iwasawa. Lectures on p-Adic L-Functions, Ann. of Math. Stud., 74. Princeton, NJ: Princeton Univ. Press, 1972.

[Kahn 93] B. Kahn. "On the Lichtenbaum-Quillen Conjecture." In Algebraic K-Theory and Algebraic Topology, edited by P. G. Goeiss and J. F. Jardine, pp. 147-166, NATO Adv. Sci. Inst. Ser. C, 407. Dordrecht: Kluwer Acad. Publ., 1993.

[Knuth 81] D. Knuth. The Art of Computer Programming, Vol. 2: Seminumerical Algorithms, Second edition. Reading, MA: Addison-Wesley Publishing Co., 1981.
[Kolster et al. 96] M. Kolster, T. Nguyen Quang Do, and V. Fleckinger. "Twisted $S$-Units, $p$-Adic Class Number Formulas, and the Lichtenbaum Conjectures." Duke Math. J. 84 (1996), 679-717.

[Kraft and Schoof 95] J. S. Kraft and R. Schoof. "Computing Iwasawa Modules of Real Quadratic Number Fields." Compositio Math. 97 (1995), 135-155.

[Kubota and Leopoldt 64] T. Kubota and H. W. Leopoldt. "Eine $p$-adische Theorie der Zetawerte, I. Einführung der $p$-adischen Dirichletschen $L$-Funktionen." J. reine angew. Math. 214/215 (1964), 328-339.

[Kurihara 92] M. Kurihara. "Some Remarks on Conjectures About Cyclotomic Fields and K-Groups of $\mathbb{Z}$." Compositio Math. 81 (1992), 223-236.

[Mazur and Wiles 84] B. Mazur and A. Wiles. "Class Fields of Abelian Extensions of $\mathbb{Q}$." Invent. Math. 76 (1984), 179-330.

[Schoof 03] R. Schoof. "Class Numbers of Real Cyclotomic Fields of Prime Conductor." Math. Comp. 72 (2003), 913-937.

[Soulé 79] C. Soulé. " $K$-théorie des anneaux d'entiers de corps de nombres et cohomologie étale." Invent. Math. 55 (1979), 251-295.

[Soulé 03] C. Soulé. "A Bound for the Torsion in the $K$ Theory of Algebraic Integers." Doc. Math. Extra Volume (2003), 761-788.

[Sumida-Takahashi 04] H. Sumida-Takahashi. "Computation of Iwasawa Invariants of Certain Real Abelian Fields." J. Number Theory 105 (2004), 235-250.

[Voevodsky 03] V. Voevodsky. "Motivic Cohomology with $\mathbb{Z} / 2$-Coefficients." Publ. Math. Inst. Hautes Études Sci. 98 (2003), 59-104.

[Washington 97] L. Washington. Introduction to Cyclotomic Fields, Second edition. Graduate Texts in Math., 83. New York: Springer-Verlag, 1997.

[Wiles 90] A. Wiles. "The Iwasawa Conjecture for Totally Real Fields." Ann. of Math. 131 (1990), 493-540.

Hiroki Sumida-Takahashi, Faculty of Integrated Arts and Sciences, Hiroshima University, Kagamiyama, Higashi-Hiroshima 739-8521, Japan (hiroki@mis.hiroshima-u.ac.jp)

Received June 15, 2004; accepted April 20, 2005. 